\title{
Importance of the Vane Exit Mach Number on the Axial
}

\section{Clearance Related Losses}

\author{
Aki Grönman', Marc H.-O. Biester ${ }^{3}$, Teemu Turunen-Saaresti ${ }^{2}$, Ahti Jaatinen-Värri² ${ }^{2}$ Jari Backman $^{2}$ \\ and Jörg R. Seume ${ }^{3}$ \\ ${ }^{1}$ Corresponding author, aki.gronman@lut.fi, School of Energy Systems, “Larjola Laboratory of \\ Turbomachinery”, Lappeenranta University of Technology, P.O. Box 20, FI-53851 Lappeenranta, Finland, \\ Telephone +35840776 7999 \\ ${ }^{2}$ School of Energy Systems, "Larjola Laboratory of Turbomachinery”, Lappeenranta University of \\ Technology P.O. Box 20, FI-53851 Lappeenranta, Finland \\ ${ }^{3}$ Institute of Turbomachinery and Fluid Dynamics, Leibniz Universität Hannover, Appelstrasse 9, 30167 \\ Hannover, Germany
}

\begin{abstract}
More efficient and physically smaller axial turbine designs are promoted to lower emissions and increase revenue. The physical size and the weight of the axial turbine can be minimised by adjusting the distance between successive stator and rotor rows. The influence of changing stator-rotor axial clearance can usually have either a positive or a negative influence on the turbine performance, and the reasons for this varying behaviour are not currently fully understood. A previous study revealed several design parameters that correlate with the efficiency curve shape. However, the effects of two parameters, namely the stator outlet Mach number and the reduced blade passing frequency, still remained unclear. Therefore, a novel approach is taken to analyse
\end{abstract}


the correlations between the two design parameters and the axial clearance efficiency curve shape. Several different turbines are analysed using data available in the literature, and also new data is presented. The study suggests that the stator outlet Mach number correlates reasonably well with the efficiency curve shape, and it was further linked to five loss mechanisms and the rotational speed. Although the unsteady interaction plays an important role in the loss share, the level of unsteadiness did not correlate with the efficiency curve shape.

Keywords: Axial turbine, axial clearance, axial gap, turbine efficiency

\section{I.Introduction}

Axial turbines are used e.g. in gas turbines, steam turbines, as well as organic Rankine cycle applications, and the improvements in turbine efficiency will contribute directly to more energy-efficient processes ${ }^{1}$. The turbine designer is often required to minimise the size and weight of the machine, yet to maximise the efficiency. One way to decrease the size of a turbine is to decrease the clearance between the turbine stator and rotor, namely the axial clearance. This decrease might, however, influence not only the efficiency of the turbine $e^{2,3}$,

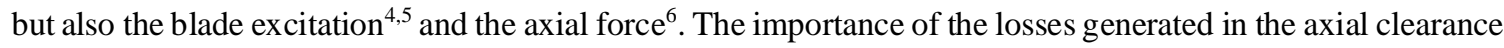
in a two-dimensional loss share was shown by Mokulys et al. ${ }^{7}$; their results indicated that these losses were the second highest after the stator profile loss.

The effect which the axial clearance has on the turbine performance can differ between turbines, and different loss mechanisms are reported to influence the curve shapes. In some studies, the efficiency decreases as the clearance increases ${ }^{3,8,9}$. However, some studies suggest a larger clearance to be more beneficial in terms of efficiency ${ }^{10,11}$. This behaviour is illustrated in Fig. 1, where the efficiency change as a function of axial clearance is given relative to the zero axial clearance for turbines studied in this article. Considering the magnitude of the efficiency change due to axial clearance, differences of even a few percentage points may be 
realised in stage efficiencies. Maximum linear correlations are plotted as solid lines. It has been suggested that the axial clearance $g / c_{\mathrm{x}}$ between 0.35 and 0.42 is a good choice to begin the design process with ${ }^{12}$ (area shown between dotted lines). This choice is also close to the common design practices ${ }^{7}$ where $g / c_{\mathrm{x}}$ is $0.3-0.4$.

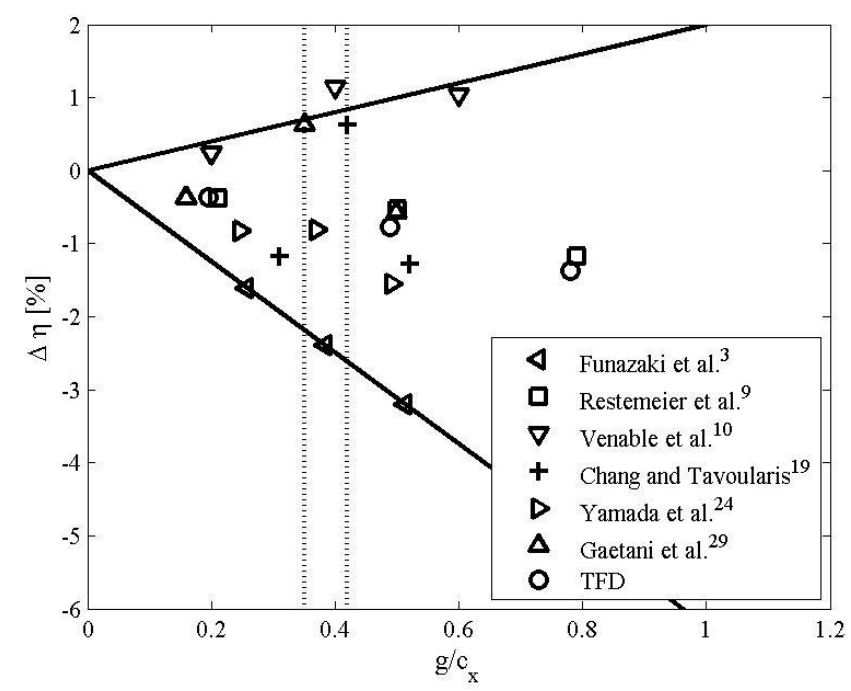

Fig. 1 Efficiency change as a function of axial clearance $g / c_{x}$

When the Mach number increases to transonic or supersonic values, the trailing edge shocks generate additional losses by interacting with the boundary layers of the neighbouring stator and also with the downstream rotor. It is also worth mentioning, that the stator wake interacts with the downstream rotor and influences the rotor boundary layer as well as the rotor wake. ${ }^{13}$ In addition, the mixing of the main flow, stator wakes, and shock waves cause losses in the stator-rotor axial clearance. As the axial clearance increases, the intensity of the trailing edge shock seems to decrease, as suggested by Denos et al. ${ }^{14}$. An example of the mixing loss evolution is plotted in Fig. 2, showing an increase of losses as the Mach number approaches unity and the 
shocks begin to influence the flow field. The losses have been calculated from the measured data with the expression given by Benner et al. ${ }^{15}$, and they form $20 \%$ of the overall mixed out losses of the cascade

$$
\frac{\Delta Y_{\text {tot }(\text { mixing })}}{Y_{\text {total }(\text { measured })}}=1-2 x / c_{x} .
$$

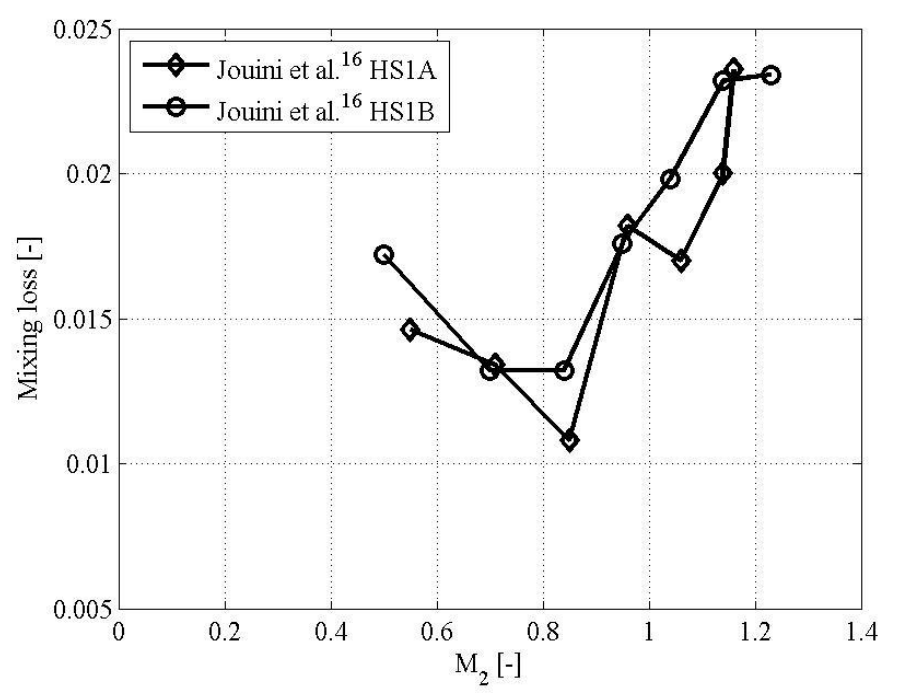

Fig. 2 Influence of outlet Mach number on mixing losses.

The losses related to the decay of stator secondary flows due to a changing Mach number also influence the flow in the axial clearance. This is illustrated in Fig. 3 where the secondary loss is presented as a function of distance from the trailing edge for different Mach numbers ${ }^{17}$. For subsonic Mach numbers, the importance of secondary losses seems to be higher than for transonic or supersonic flows. It is noticeable that in the range of typical axial clearance $\left(x / c_{\mathrm{x}}=1.3-1.5\right)$ there is a secondary loss reduction visible in every Mach number. The study of Perdichizzi ${ }^{17}$ also illustrated the influence of the Mach number on the secondary flow structures, which will further have influence on the interaction with the rotor blade and secondary flows. The increasing Mach 
number tends to push the passage vortex towards the endwall region, but also the shock waves can interact with the endwall boundary layer. It was shown that by increasing the distance from the trailing edge at supersonic Mach numbers, the mixing process leads to a more uniform loss distribution. The importance of shed and corner vortices is also greater compared to the passage vortex in loss generation when the Mach number is increased. The positions of the associated loss cores are also influenced by the Mach number. This will most likely also have an influence on the interaction between the secondary flows and the rotor, but also the interaction with rotor secondary flow structures can be influenced. The importance of secondary flows is also suggested to become smaller when the three dimensional effects from shock waves begin to influence at higher supersonic Mach numbers.

Gaetani et al. ${ }^{18}$ have identified a wake like behavior of the stator hub passage vortex showing bowing, stretching and chopping of the vortex. The described interaction with the rotor blade further influenced the rotor hub passage vortex development. This interaction was still found to influence the rotor outlet flow field even when the axial clearance was equal to stator axial chord. They concluded that the stator flow mostly affects the rotor hub region, while the channel above $60 \%$ of the span is almost unaffected due to mixing out of the vortices and wake. The smaller axial clearance seems to lead to more intense interaction between the stator and rotor, including both wake and vortex interaction.

As mentioned by Gaetani et al. ${ }^{18}$, the main aspects of the two-dimensional interaction between the stator and rotor are related to the potential field and the wake. Funazaki et al. ${ }^{13}$ have showed that the stator wake interacts with the downstream rotor and influences the rotor boundary layer but also the rotor wake. The mixing of the wake is strongly dependent on the axial clearance and the wake propagation depends mostly on the blade discharge angle. The potential field, however, is a more complex function of blade loading, loading position (front, aft, mid), suction side curvature, etc. The influence of the potential field is shown in a study by Mokulys 
et al. ${ }^{7}$ indicating that major reductions in the axial clearance losses are possible by changing the rotor loading. This further indicates that different blade loading may lead to changes in the axial clearance-efficiency curve shape.

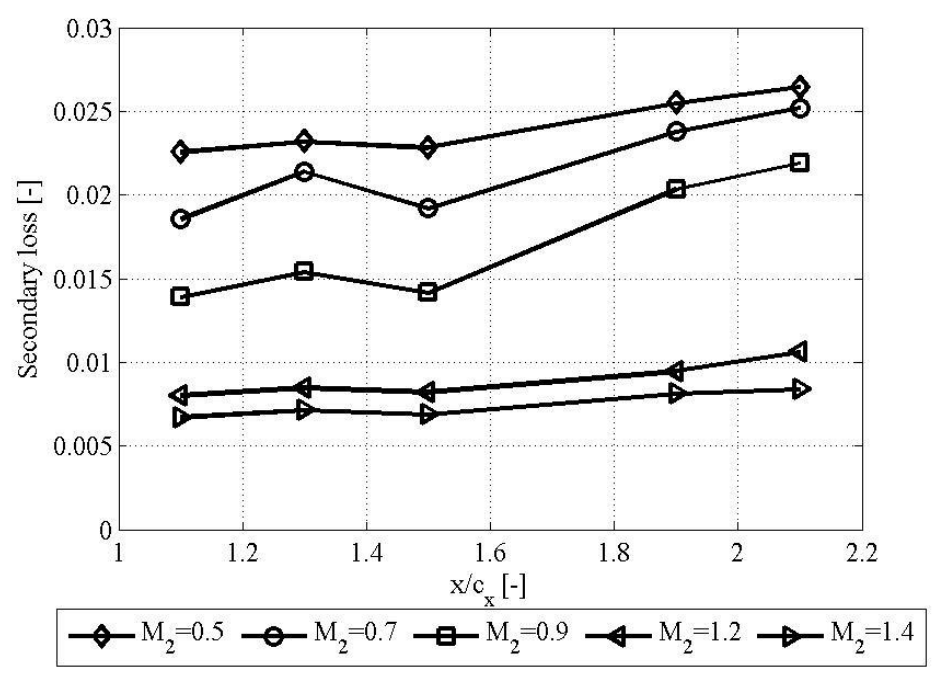

Fig. 3 Decay of the secondary losses as a function of the distance from the trailing edge ${ }^{17}$.

Axial clearance also influences the flow unsteadiness in the rotor. Several studies have found the rotor pressure fluctuation to be smaller when the clearance is increased, and this behaviour does not seem to require the presence of shock waves ${ }^{8,19-21}$. Due to changes in pressure fluctuation, also unsteady work ${ }^{22,23}$ and the enthalpy change at the rotor inlet are influenced when the axial clearance changes.

Although the unsteady stator-rotor interaction is an important contributor to the overall turbine performance, it is a complex mechanism and it cannot be said whether the weaker interaction is always more beneficial or whether the stronger interaction should be the aim, as proposed by Rose et al. ${ }^{23}$. The positivity or negativity of the stator-rotor interaction can also vary from hub to shroud, as shown by Yamada et al. ${ }^{24}$ and Gaetani et al. ${ }^{2,18}$ 
In addition, Gaetani et al. ${ }^{25}$ reported that the wake-induced interaction decreased the efficiency with larger clearances and the vortex-induced interaction with small clearances.

A previous study ${ }^{12}$ concluded that there are five main loss sources that are affected by the axial clearance: 1. stator wake mixing with the main flow, 2. unsteady interaction between the stator wake and rotor blade, 3 . changing rotor incidence, 4 . endwall boundary layer losses and 5. stator trailing edge shock related losses. The study was able to link four loss sources to the correlating design parameters, leaving only stator trailing edge shock related losses out of the analysis due to a lack of data. It was also suggested that the unsteady stator-rotor interaction should be included directly in the analysis. The current study has also highlighted the importance of the stator secondary flow structures, their decay and interaction with the rotor blade and its flow structures as an important loss contributor that is affected by the axial clearance.

This study aims to shed light on the correlations of the remaining two non-dimensional parameters by applying linear correlation analysis used in the previous article ${ }^{12}$ and an earlier on by Turunen-Saaresti and Jaatinen $^{26}$. First, the geometry and the numerical method used for the new turbine data are presented. Second, data available in the literature and the linear correlation analysis method are described. Third, the correlations of the stator outlet Mach number and reduced blade passing frequency (RBPF) are studied by analysing the influence of axial clearance on the loss mechanisms. Furthermore, suggestions for mechanisms behind the different curve shapes are given and the reasons for similarities between the stator outlet Mach number and the rotational speed curves are proposed.

\section{II.Numerical methods and TFD turbine rig}

The results labelled as 'TFD' have been obtained from numerical computations using the unsteady Reynolds-averaged Navier Stokes (URANS) flow solver for structured and unstructured grids referred to as 
TRACE (Turbomachinery Research Aerodynamics Computational Environment). The leading development of TRACE is done by the Institute of Propulsion Technology of the German Aerospace Center (DLR). The $k-\omega$ turbulence model is used to account for turbulent effects. A correlation-based transition model calibrated to provide good results for natural-, bypass-, separation- and wake-induced transition is employed at the suctionside of the blades and vanes. All other walls are considered to be fully turbulent. All walls have a $y^{+}$-value of $y^{+}<1$ and a corresponding approach is used to capture effects of major importance occurring within the boundary layer. The results of a grid dependency study and further information on the grid, geometry as well as spatial and temporal discretisation is presented $\mathrm{in}^{27}$. For the computations, a mesh with sufficiently fine grid-resolution is used.

The computations represent a 11/2-stage turbine rig located at the Institute of Turbomachinery and Fluid Dynamics of the Leibniz Universität Hannover, Germany. The stage loading $\Delta h_{t} / U^{2}$ at the design operatingpoint amounts approximately to 1.95 , and the corresponding flow coefficient is $C_{\mathrm{x}} / U \approx 0.6$. The overall blade loading is comparably small due to a high blade count in the second stator and rotor row. In the midspan region, however, the blade loading is comparable to the blade loading of low-pressure aircraft turbines. Furthermore, in this region the flow-field is characterised by a 2D flow-field. The Reynolds number based on the exit conditions and chord is comparably high $(\approx 600.000)$, and boundary-layer separation does not occur. Simulations are performed for three different axial clearances that correspond to $0.2 c_{\mathrm{x}}, 0.5 c_{\mathrm{x}}$ and $0.8 c_{\mathrm{x}}$. Recent studies on that rig have shown that cavity flow strongly influences the slope in efficiency due to changes in the axial clearance ${ }^{28}$. In order to obtain an isolated view neglecting the named effect, computations do not include cavities such as labyrinth-seals. Particularly for small axial clearances of $0.2 c_{\mathrm{x}}$, recent investigations have shown that clocking has a strong influence on the efficiency of the turbine. The presented slope accounts for that effect by providing an average value determined by simulations performed at different clocking positions. 


\section{III.Analysed data and linear correlation method}

Several turbine geometries varying in their design values were analysed in order to study the effects of the stator outlet Mach number and reduced blade passing frequency. These test cases are described in Table 1, presenting values in design operating conditions. Most of the data is taken from the referred publications or is calculated/estimated from the published data, but also new data is presented from the current TFD test case. Data gathered from the literature was obtained by measurements and unsteady numerical simulations.

To ensure that the data between different test cases is comparable, the following discussion concentrates on evaluating the comparability of the data sets. When examining the possible reasons that make the comparison between turbines challenging, the distances from the inlet plane to the first stator and from the last blade or vane to the outlet plane vary between most the cases. At the inlet, it is likely that this varying distance has a negligible influence on the results. However, at the outlet the mixing process depends more on the distance from the last blade/vane trailing edge. Benner et al. ${ }^{15}$ assume that fully mixed out losses are reasonably estimated at the midspan when the measurement locations were at least $20 \%$ of axial chord length downstream of the trailing edge. All of the examined turbines fulfil this requirement. Another noticeable issue is the number of stages. Five of the test cases are single-stage turbines and two are 1.5 stage machines. Restemeier et al. ${ }^{9}$ suggest, that the second stator and the preceding axial clearance only has a minimal influence on the loss differences between the cases with different axial clearances. With this assumption, a direct comparison of single and 1.5 stage turbines is reasonable and a possible small error does not have a notable influence on the accuracy of the current analysis.

It is noteworthy that the direction and magnitude of the trailing edge shocks may variate from hub to shroud due to radial equilibrium. ${ }^{29}$ Stronger shocks are expected at the hub and weaker at the shroud. The change in the shock angle also influences the interaction between the rotor blade and shock. This means that with some 
designs there is the possibility that the flow is subsonic at some part of the span and elsewhere transonic and the interaction mechanism is different from a fully transonic case. It is, however, believed that the meanline values used here are representative to highlight the major phenomena inside a turbine.

Table 1 Data for linear correlation analysis

\begin{tabular}{|c|c|c|c|}
\hline Reference & RBPF & $\bar{M} M_{2}$ & "Meas./URANS \\
\hline Funazaki et al. $^{3}$ & 4.32 & - & Meas. \\
\hline Restemeier et al. ${ }^{9}$ & 1.31 & 0.45 & Meas. \\
\hline Venable et al. ${ }^{10}$ & 4.84 & 1.10 & URANS \\
\hline Chang and Tavoularis ${ }^{19}$ & - & 1.18 & URANS \\
\hline Yamada et al. $^{24}$ & 3.99 & 0.18 & Meas. \\
\hline Gaetani et al. ${ }^{25}$ & 2.30 & 0.55 & Meas. \\
\hline TFD & 2.37 & 0.50 & URANS \\
\hline
\end{tabular}

The analysis began by assuming a linear correlation in all studied cases for the efficiency as a function of stator-rotor axial clearance in Fig. 1. The coefficient of determination $R^{2}$ was then used to evaluate the correlation of each fit separately. Table 2 presents the results of this analysis, suggesting relatively linear correlations for most of the turbines. A positive (or close to zero) value in the slope of efficiency curve $k$ denotes a turbine which has increasing efficiency when the axial clearance is increased from its minimum, whereas a negative value denotes a turbine that has the best efficiency outside the minimum clearance. The weakness of the method used is that some of the turbines represent non-linear behaviour (see Fig. 1.), and it can be questioned whether it is captured in the analysis. Therefore, extra care is taken to look behind the correlations in the analysis to avoid miss-interpretation of the results due to a lack of accuracy. It is also worth mentioning, that parallel to 
the presented results, efforts were made to fit the data non-linearly. That study did not produce any further understanding in addition to the results presented here.

Table 2 Coefficient of determination for the slope in efficiency based on Fig. 1

\begin{tabular}{lccc}
\hline \hline Reference & $k$ & $R^{2}$ & $n$ \\
\hline Funazaki et al. $^{3}$ & -6.24 & 1.00 & 3 \\
Restemeier et al. $^{9}$ & -1.39 & 0.89 & 3 \\
Venable et al. $^{10}$ & 2.00 & 0.66 & 3 \\
Chang and Tavoularis $^{19}$ & 0.35 & 0.01 & 3 \\
Yamada et al. $^{24}$ & -2.89 & 0.74 & 3 \\
Gaetani et al. $^{25}$ & -0.33 & 0.01 & 3 \\
TFD & -1.71 & 0.99 & 3 \\
\hline \hline
\end{tabular}

As the next step, the slope in efficiency values from Tab. 2 were assumed to represent the efficiency curve shapes of different turbines. After this assumption, the correlations between the two parameters from Tab. 1 and the slopes of efficiency were evaluated.

\section{IV.Correlations of stator outlet Mach number and reduced blade passing frequency}

Figure 4 presents the correlation between the stator outlet Mach number and the slope in efficiency. A good linear correlation with the coefficient of determination of 0.81 is found, and when only subsonic designs are considered, $R^{2}$ is 0.82 . The indicated small change in the curve slope at Mach numbers above unity can be explained by the presence of stator trailing edge shock waves. As the study of Denos et al. ${ }^{14}$ suggests, the shock wave magnitude decreases with the increasing axial clearance. This behaviour means that the shock waves 
interacting with the rotor blades are weaker and the losses in the rotor due to shock-rotor interaction are lower when the axial clearance increases. The described mechanism also explains why the two transonic turbines in Fig. 1 experience increasing efficiency when the axial clearance is increased from its minimum value. However, a further increase of axial clearance does not lead to improved performance in the two transonic turbines, and therefore, the driving loss mechanism with larger clearances has to be something other than the shock waverotor interaction.

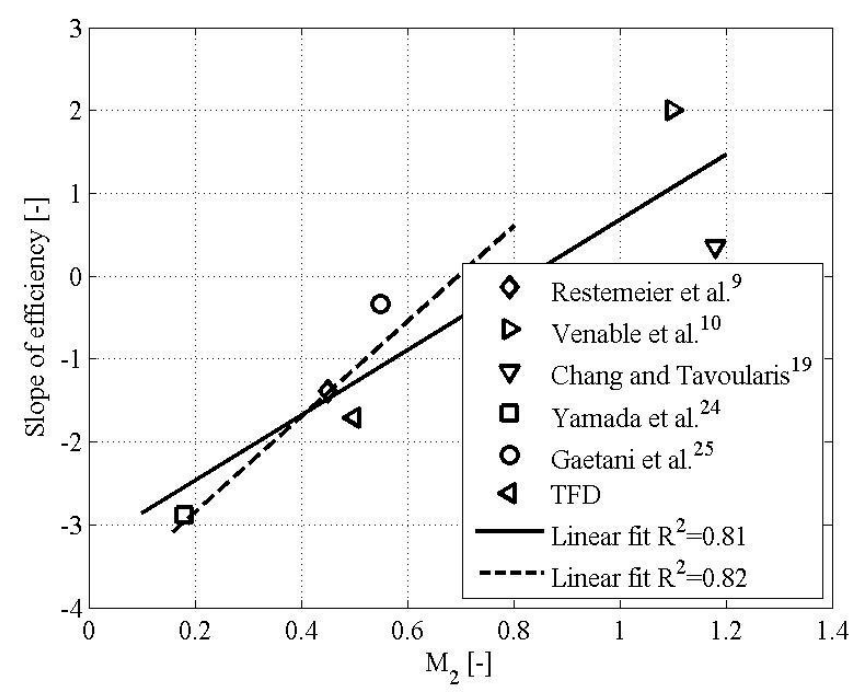

Fig. 4 Correlation between $M_{2}$ and the slope in efficiency.

One explanation for the drop in the transonic turbine efficiency at a larger clearance is the increasing mixing losses, and another is the changes in stator-rotor interaction, as explained by Busby et al. ${ }^{30}$. The influence of mixing losses is quite straightforward: as the distance from the stator trailing edge increases, the losses due to mixing also increase. The influence of shock waves on the mixing loss is also clearly visible in Fig. 2, which shows that the overall mixing losses increase rapidly at Mach numbers approaching unity. 
At subsonic Mach numbers, the mixing losses seem to have even higher importance at least with low pressure turbine designs. Muth and Niehuis ${ }^{31}$ report that at low Reynolds numbers $(\operatorname{Re}=40000)$, the mixing loss can contribute $50 \%$ of the overall loss share in a cascade, and at higher Reynolds numbers, they will drop to $35 \%(\mathrm{Re}=400000)$. If the operating conditions are kept constant or nearly constant, the increase of the Reynolds number also increases the Mach number, and the conclusions of Muth and Niehuis ${ }^{31}$ are valid in the current analysis.

The Mach number is also connected to the endwall boundary layer growth and its interaction with the rotor at hub and tip areas. A simple boundary layer thickness analysis over a flat plate shows that an increase of the Reynolds number decreases the thickness of a boundary layer. If the assumptions for constant or nearly constant operating conditions are met, at transonic flows the endwall boundary layers are thinner than they are at subsonic velocities, and therefore, the losses related to thicker viscous boundary layer interaction should also be more important at subsonic designs.

Based on the analysis, the importance of mixing and endwall boundary layer interaction losses can be more significant at low stator outlet Mach number levels than at higher Mach numbers. If the mixing and endwall boundary layer losses are the dominating mechanisms, a drop in the efficiency would be realised with increasing clearance. However, the curve shape in Fig. 4 indicates the opposite behaviour where the slope of efficiency can also be positive or the maximum efficiency is reached outside the minimum clearance. This change in the slope gives an indication that the unsteady stator-rotor interaction might play a larger role in the overall loss share as the Mach number increases.

An effort to shed some light on the unsteady mechanisms behind the different curve shapes is made by combining understanding from the cascade measurements with the current understanding about the unsteady interaction mechanisms with different axial clearances. As was shown by Perdichizzi ${ }^{17}$, the increase of the stator 
outlet Mach number makes the shed and corner vortex more important than the passage vortex is. On the other hand, Gaetani et al. ${ }^{18}$ showed that with very large axial clearance $\left(g / c_{\mathrm{x}}=1\right)$ the stator hub shed vortex is still interacting with the rotor hub passage vortex but other stator structures are mixed out after the rotor. This indicates, that as the Mach number increases and the maximum efficiency is reached with respect to the axial clearance, the interaction between the shed and corner vortex with the rotor blade and its secondary structures could start to produce additional (to mixing and end wall boundary layer) losses which lead to a drop of efficiency. Although the interaction of the potential field and wake with the following rotor blade seems to have a great influence on the losses as the axial clearance changes ${ }^{18,25}$ it is yet unclear what kind of influence the stator outlet Mach number has on this loss mechanism. The study by Perdichizzi ${ }^{17}$ suggested that the spanwise position of the two-dimensional flow is influenced by the Mach number, which indicates that the influence on the interaction exists.

The found Mach number correlation can be also connected to the correlation between the rotational speed and the slope in efficiency, to put it into an even larger context. Chang and Tavoularis ${ }^{19}$ conclude in their literature review that the efficiency trend as a function of axial clearance in high pressure turbines depends on rotational speed. Figure 5 illustrates this behaviour for several turbine geometries (including both high and lowpressure designs), and it is noticeable that the curve trend is somewhat similar to the Mach number trend with one outlier ${ }^{19}$. Note that the $R^{2}$ value is calculated without the outlier, which is not shown in the figure. The outlier is most likely due to the markedly higher speed of sound compared to the other studied cases.

To explain the similarity between Figs. 4 and 5, the velocity triangles of the different designs and the operating conditions are examined. Figure 6 presents the stator outlet/rotor inlet velocity triangles for five turbines at midspan. It is common knowledge that, the peripheral speed is a function of the rotational speed and the radius. In the five turbines shown in Fig. 6, the radii in different designs are reasonably close to each other, 
and therefore, the differences in velocity triangles result mostly from the rotational speed and mass flow. In addition, the relative flow angles do not markedly differ between the designs, which means that the increasing peripheral velocity (or rotational speed) will also increase the absolute velocity. On the other hand, the Mach number depends on the absolute flow velocity and static temperature (fluid is air for the five turbines in Fig. 6), and the temperature and pressure ratio levels especially below Mach 1 are close to each other. Therefore, the absolute flow velocity almost linearly represents the stator outlet Mach numbers.

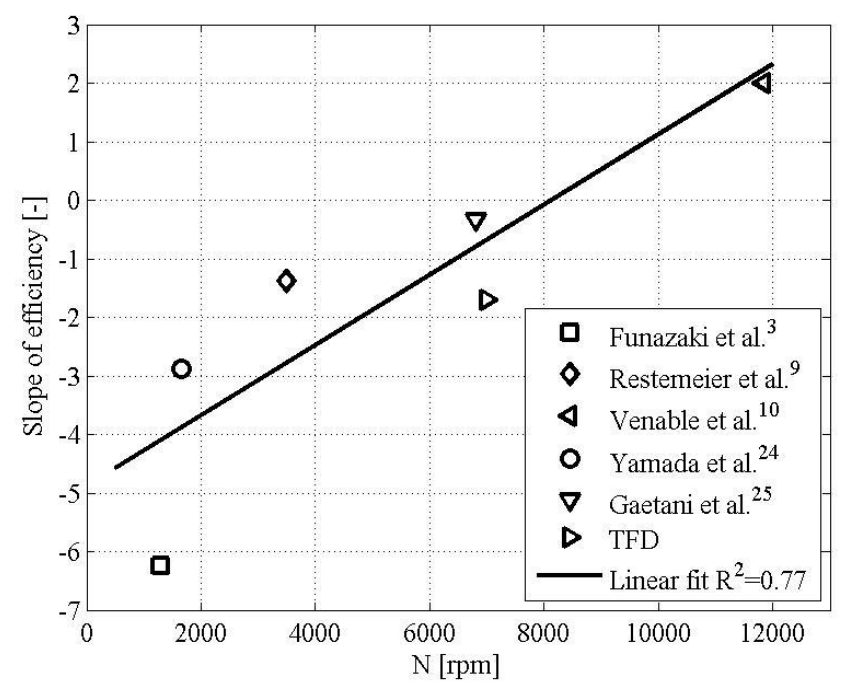

Fig. 5 Correlation between the rotational speed and the slope in efficiency. 


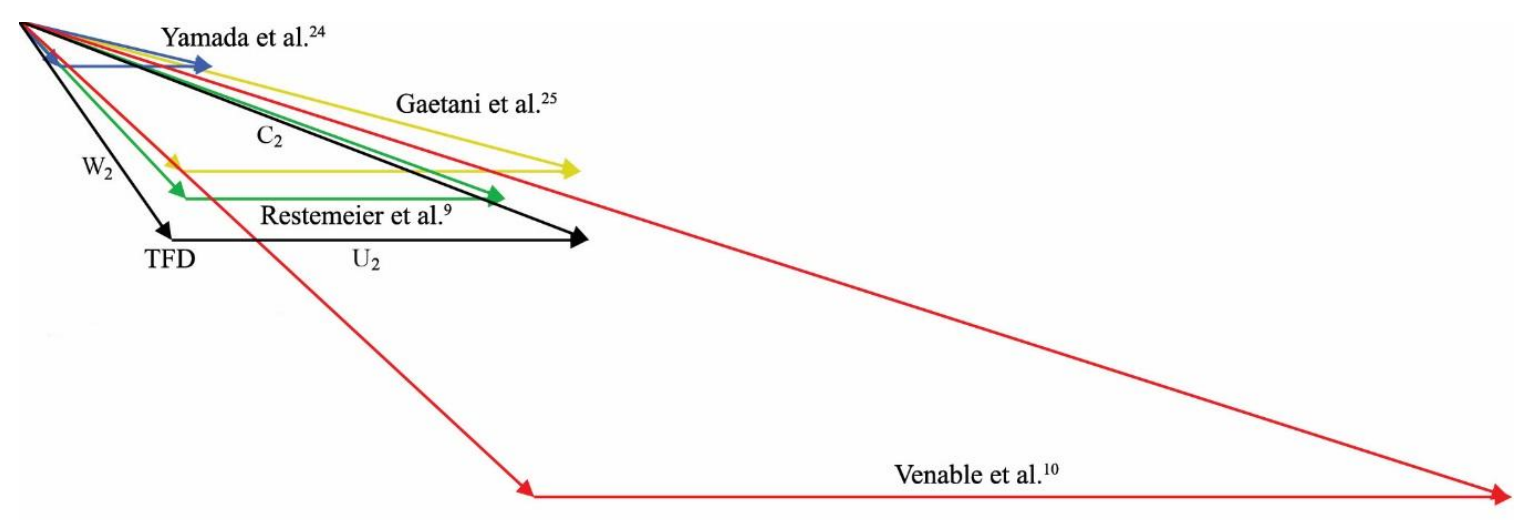

Fig. 6 Midspan velocity triangles at the stator outlet/rotor inlet.

The flow unsteadiness has been shown to be influenced by the axial clearance variation by many authors ${ }^{8,19-}$ ${ }^{21}$. Figure 7 illustrates how the magnitude of unsteadiness influences the shape of the efficiency curves. As can be seen, there is no clear correlation between the reduced blade passing frequency and the slope of efficiency for the studied turbine geometries. Two of the cases have an RBPF close to four but the slopes of efficiency are drastically negative (an increase in the axial clearance will decrease the efficiency). Other cases seem to present a weak trend where the increase of the RBPF will lead to designs which will result in higher efficiency when the axial clearance is increased from its minimum value; the data is, however, too limited to draw a strong conclusion.

The observed lack of correlation raises the question of why the RBPF does not correlate with the slope of efficiency although the unsteady interaction is clearly important. One answer to this is that the level of unsteadiness itself is not the driving mechanism for different axial clearance effects. Another explanation is that the RBPF mostly influences the rotor efficiency and, therefore, a weak impact on the mixing of stator structures in the axial clearance is expected. The stator outlet Mach number, however, seems to have an influence on 
several loss mechanisms and may be one of the most important design parameters influencing the different efficiency curve shapes.

To highlight the importance of the $M_{2}$ over RBPF, a combined analysis was made to fit the $M_{2}$ and RBPF simultaneously with the efficiency curve shape by using linear least squares fitting. Although the identified correlation is strong, $R^{2}=0.95$, the influence of the RBPF is less than $1 \%$ of the one that the stator outlet Mach number has $\left(k=-3.774+5.418 M_{2}-0.034 \mathrm{RBPF}\right)$. This finding is a clear indication that the influence of the RBPF as a correlating parameter is negligible and supports the results of studying the correlations separately.

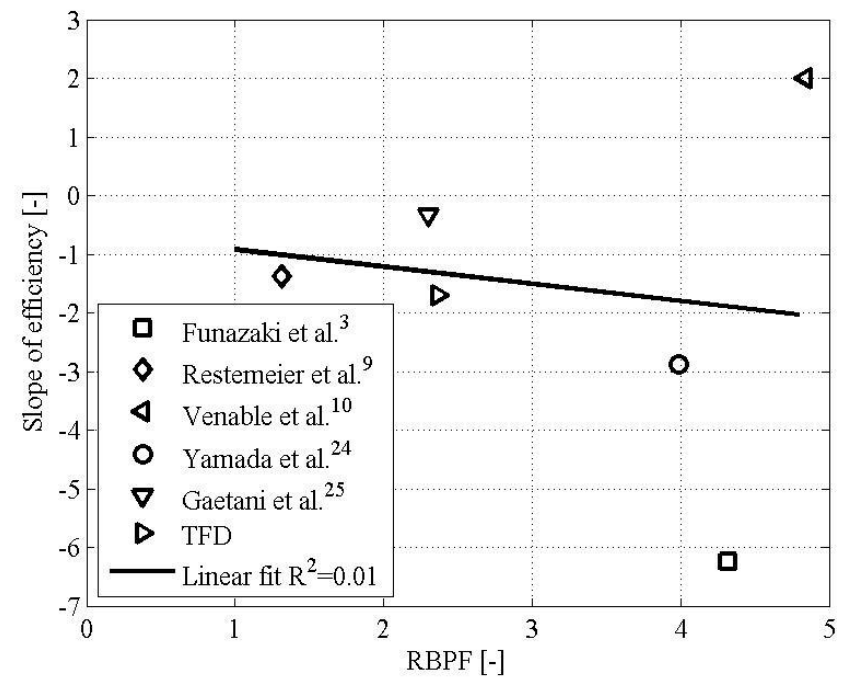

Fig. 7 Correlation between reduced blade passing frequency and the slope in efficiency.

\section{V.Conclusions}

The study conducted suggests that there is a correlation between the stator outlet Mach number and the slope of the efficiency curve. The increasing Mach number changes the efficiency curve shape, as a function of 
stator-rotor axial clearance, in a direction where increasing the clearance from its minimum value results in improved efficiency.

The stator outlet Mach number was linked to five loss mechanisms: stator wake mixing with the main flow, unsteady interaction between the stator wake and rotor blade, endwall boundary layer losses, stator trailing edge shock related losses and the stator secondary flow structures, their decay and interaction with the rotor blade and its flow structures. The similarities between the rotational speed and the Mach number curve shapes were also found to result from reasonably similar temperature and pressure levels and flow angles between the studied turbines.

It was also found that the reduced blade passing frequency does not correlate with the shape of the efficiency curve, although the unsteady stator-rotor interaction plays an important role in the curve shape. The influence of the unsteady interaction also seems to be positive in many of the studied turbines when the axial clearance increases, causing the optimal clearance to be other than the minimum value.

The results may have a significant influence on the future development of a correlation that can predict the influence of changing axial clearance on the turbine performance. The stator outlet Mach number will play an important role in the correlation, since it will influence five of the six loss mechanisms. Initial plans have been made to develop the correlation in future studies together with quantifying the share of each loss mechanism from the total loss budget.

Considering the application of the results obtained on multi-stage turbines, the change of axial clearance might have a similar influence on losses regardless of whether stator-rotor or rotor-stator clearance is considered. This behaviour can be justified if the driving loss mechanisms remain reasonably similar despite the considered clearance position being before or after the stator/rotor. Support for the given suggestion can be found from the transonic axial compressor study of $\mathrm{Li}$ and $\mathrm{He}^{32}$, where the compressor maximum efficiency 
was found outside the minimum clearance when the rotor-stator clearance was changed. The observed performance change is in line with the transonic turbine studies ${ }^{10,19}$. However, to be certain, more research is required on multi-stage turbines.

\section{Acknowledgments}

The authors gratefully acknowledge the substantial contribution of the DLR Institute of Propulsion Technology providing the TRACE. Additionally, the authors thank MTU Aero Engines for the permission to publish this work. Furthermore, the authors thank the Regional Computing Center for Lower-Saxony (RRZN) for the computational resources provided. The CFD results from TFD turbine rig presented in this paper have been obtained from research conducted within the fourth Aeronautical Research Program of the German Ministry of Economics.

\section{References}

1. Wisler DC. The technical and economic relevance of understanding boundary layer transition in gas turbine engines. In: Proceedings of 1997 workshop on boundary layer transition in turbomachines, Syracuse, New York, September 7-10 1997, NASA/CP-1998-206958, pp. 53-64.

2. Gaetani P, Persico G, Dossena V and Osnaghi C. Investigation of the flow field in a HP turbine stage for two stator-rotor axial clearances - Part I: Three-dimensional time-averaged flow field. J Turbomachinery 2007; 129: 572-579.

3. Funazaki K, Yamada K, Kikuchi M and Sato H. Experimental studies on aerodynamic performance and unsteady flow behaviors of a single turbine stage with variable rotor-stator axial clearance: Comparisons 
with time-accurate numerical simulation. In: Proceedings of ASME Turbo Expo, Montreal, Canada, 14-17 May 2007, paper no. GT2007-27670,pp. 703-711. New York: ASME.

4. Jöcker M. Numerical investigation of the aerodynamic vibration excitation of high-pressure turbine rotors. Doctoral Thesis, Royal Institute of Technology, Stockholm, Sweden, 2002.

5. Biester MH-O, Guendogdu Y and Seume JR. Clocking effects on surface-pressure fluctuations for variable axial-gaps. In: 21st AIAA Computational Fluid Dynamics Conference, San Diego, California, 24-27 June, 2013.

6. Baagherzadeh Hushmandi $\mathrm{N}$ and Fransson TH. Effects of multiblocking and axial clearance distance on performance of partial admission turbines: A numerical analysis. J Turbomachinery 2011; 133: 031028.

7. Mokulys T, Congiu F, Rose MG and Abhari RS. Unsteady numerical investigation of the effect of wakes with eddy shedding in different axial turbine aerofoils. Proc IMechE Part A: J Power Energy 2009; 223: 1001-1016.

8. Park J, Choi M and Baek J. Effects of axial clearance on unsteady secondary flow in one-stage axial turbine. Int J Turbo Jet Eng 2003; 20: 315-333.

9. Restemeier M, Jeschke P, Guendogdu Y and Gier J. Numerical and experimental analysis of the effect of variable blade row spacing in a subsonic axial turbine. J Turbomachinery 2013; 135: 021031.

10. Venable BL, Delaney RA, Busby JA, Davis, RL, Dorney DJ, Dunn MG, Haldeman CW and Abhari RS. Influence of vane-blade spacing on transonic turbine stage aerodynamics: Part I - Time-averaged data and analysis. J Turbomachinery 1999; 121: 663-672.

11. van de Wall AG, Kadambi JR and Adamczyk JJ. A transport model for the deterministic stresses associated with turbomachinery blade row interactions. J Turbomachinery 2000; 122: 593-603. 
12. Grönman A, Turunen-Saaresti T, Röyttä P and Jaatinen-Värri A. Influence of the axial turbine design parameters on the stator-rotor axial clearance losses. Proc IMechE Part A: J Power Energy 2014; $228: 482$ 490.

13. Funazaki, K., Sasaki, Y. and Tanuma, T. Experimental studies on unsteady aerodynamic loss of a high pressure turbine. In: Proceedings of ASME International Gas Turbine and Aeroengine Congress and Exhibition, Orlando, Florida, 2-5 June 1997, paper no. 97-GT-52. New York: ASME.

14. Dénos R, Arts T, Paniagua G, Michelassi F and Martelli F. Investigation of the unsteady rotor aerodynamics in a transonic turbine stage. J Turbomachinery 2001; 123: 81-89.

15. Benner MW, Sjolander SA and Moustapha SH. An empirical prediction method for secondary losses in turbines-Part II: A new secondary loss correlation. J Turbomachinery 2006; 128: 281-291.

16. Jouini DBM, Sjolander SA and Moustapha SH. Midspan flow-field measurements for two transonic linear turbine cascades at off-design conditions. J Turbomachinery 2002; 124: 176-186.

17. Perdichizzi, A. Mach number effects on secondary flow development downstream of a transonic cascade. $J$ Turbomachinery 1990; 112: 643-651.

18. Gaetani P, Persico G, Dossena V and Osnaghi C. Investigation of the flow field in a HP turbine stage for two stator-rotor axial clearances - Part II: Unsteady flow field. J Turbomachinery 2007; 129: 580-590.

19. Chang D and Tavoularis S. Effect of the axial spacing between vanes and blades on a transonic gas turbine performance and blade loading. Int J Turbo Jet Eng 2013; 30: 15-31.

20. Griffin LW and Dorney DJ. Simulations of the unsteady flow through the Fastrac supersonic turbine. $J$ Turbomachinery 2000; 122: 225-233.

21. Ummiti MB, Sitaram N and Prasad BVSSS. Computational investigation of effect of axial spacing on blade row interaction in a 1.5 stage axial flow turbine. Eng Appl Comput Fluid Mech 2009; 3: 56-70. 
22. Rose, MG, Jenny, P, Gier, J and Abhari, RS. Experimentally observed unsteady work at inlet to and exit from an axial flow turbine rotor. J Turbomachinery 2013; 135: 061017.

23. Rose M, Schüpbach $\mathrm{P}$ and Mansour M. The thermodynamics of wake blade interaction in axial flow turbines: Combined experimental and computational study. J Turbomachinery 2013; 135: 031015.

24. Yamada K, Funazaki K, Kikuchi M and Sato H. Influences of axial clearance between blade rows on secondary flows and aerodynamic performance in a turbine stage. In: Proceedings of the ASME Turbo Expo, Orlando, Florida, 8-12 June 2009, paper no. GT2009-59855, pp. 1039-1049. New York: ASME.

25. Gaetani P, Persico G and Osnaghi C. Effects of axial clearance on the vane-rotor interaction in a low aspect ratio turbine stage. J Prop Power 2010; 26: 325-334.

26. Turunen-Saaresti $\mathrm{T}$ and Jaatinen A. Influence of the different design parameters to the centrifugal compressor tip clearance loss. J Turbomachinery 2013; 135: 011017.

27. Biester MH-O, Henke M, Guendogdu Y, Engel K and Seume JR. Unsteady wake-blade Interaction: a correlation between surface pressure fluctuations and loss generation. In: Proceedings of ASME Turbo Expo, Copenhagen, Denmark, 11-15 June 2012, paper no. GT2012-69616. New York: ASME.

28. Biester MH-O, Wiegmann F, Guendogdu Y and Seume JR. Time-resolved numerical study of axial gap effects on labyrinth-seal leakage and secondary flow in a LP turbine. In: Proceedings of ASME Turbo Expo, San Antonio, Texas, 3-7 June 2013, paper no. GT2013-95628. New York: ASME.

29. de la Loma, A., Paniagua, G., Verrastro, D. and Adami, P. Transonic turbine heat transfer investigation in presence of strong shocks. J Turbomachinery 2008; 130: 031019.

30. Busby JA, Davis RL, Dorney DJ, Dunn MG, Haldeman CW, Abhari RS, Venable BL and Delaney RA. Influence of vane-blade spacing on transonic turbine stage aerodynamics: Part II - Time-resolved data and analysis. J Turbomachinery 1999; 121: 673-682. 
31. Muth B and Niehuis R. Axial loss development in low pressure turbine cascades. J Turbomachinery 2013; 135: 041024.

32. Li HD and He L. Toward intra-row gap optimization for one and half stage transonic compressor. $J$ Turbomachinery 2005; 127: 589-598.

\section{Appendix}

Notation

$b \quad$ rotor axial chord $[\mathrm{m}]$

BPP blade passing period [s]

C absolute flow velocity $[\mathrm{m} / \mathrm{s}]$

$C_{\mathrm{x}} \quad$ axial flow velocity $[\mathrm{m} / \mathrm{s}]$

$c_{\mathrm{x}} \quad$ stator axial chord [m]

$g \quad$ stator-rotor axial clearance [m]

$\Delta h_{\mathrm{t}} \quad$ change of total enthalpy $[\mathrm{J} / \mathrm{kg}]$

$k \quad$ curve slope [-]

$M \quad$ Mach number [-]

$N \quad$ number of data points [-]

$N \quad$ rotational speed [rpm]

$R^{2} \quad$ coefficient of determination [-]

$\mathrm{RBPF}$ reduced blade passing frequency, $b /\left(\mathrm{BPP} \cdot C_{\mathrm{x}}\right),[-]$

$U \quad$ peripheral speed $[\mathrm{m} / \mathrm{s}]$

$W \quad$ relative flow velocity $[\mathrm{m} / \mathrm{s}]$ 


\footnotetext{
$x \quad$ distance from the trailing edge [m]

$y^{+} \quad$ non-dimensional wall distance [-]

$Y \quad$ total pressure loss [-]

$\Delta \eta \quad$ change of efficiency [\%]
}

Subscripts

2 stator outlet, rotor inlet 